\title{
Two-way deterministic quantum key distribution against detector-side-channel attacks
}

\author{
Hua Lu, ${ }^{1,2, *}$ Chi-Hang Fred Fung, ${ }^{3, \dagger}$ and Qing-yu Cai ${ }^{2, \ddagger}$ \\ ${ }^{1}$ Department of Mathematics and Physics, Hubei University of Technology, Wuhan 430068, China \\ ${ }^{2}$ State Key Laboratory of Magnetic Resonances and Atomic and Molecular Physics, Wuhan Institute of Physics and Mathematics, \\ Chinese Academy of Sciences, Wuhan 430071, China \\ ${ }^{3}$ Department of Physics and Center of Theoretical and Computational Physics, University of Hong Kong, Pokfulam Road, Hong Kong
}

(Received 11 July 2013; published 9 October 2013)

\begin{abstract}
In a two-way deterministic quantum key distribution (DQKD) protocol, Bob randomly prepares qubits in one of four states and sends them to Alice. To encode a bit, Alice performs an operation on each received qubit and returns it to Bob. Bob then measures the backward qubits to learn about Alice's operations and hence the key bits. Recently, we proved the unconditional security of the final key of this protocol in the ideal device setting. In this paper, we prove that two-way DQKD protocols are immune to all detector-side-channel attacks at Bob's side, while we assume ideal detectors at Alice's side for error testing. Our result represents a step forward in making DQKD protocols secure against general detector-side-channel attacks.
\end{abstract}

DOI: 10.1103/PhysRevA.88.044302

PACS number(s): 03.67.Dd, 03.67.Hk, 03.67.Ac

\section{INTRODUCTION}

Quantum cryptography, also called quantum key distribution $(\mathrm{QKD})$, allows two remote parties, usually called Alice and Bob, to establish a secret key that can be used to transmit a secret message with a classical one-time pad scheme. After the pioneering work presented by Bennett and Brassard in 1984 (BB84), the unconditional security of the BB84 protocol was proven with the assumption of ideal settings [1-4]. In practice, the devices of QKD are imperfect, the eavesdropper, called Eve, can exploit the imperfections of the devices to gain partial or full information about the key bits [5,6]. It has been shown that Eve may take advantage of the nonzero multiphoton emission probability of Alice's laser source and the loss of quantum channel, by using the photon-number-splitting (PNS) attack [7-10]. Decoy state QKD has been proposed to beat Eve's PNS attacks, which can substantially increase the secure key distribution distance [11-13].

An important part of the QKD system is the measurement devices. In experiments, the detection efficiency of the practical single-photon detectors (SPDs) is low and, in most cases, the two detectors are asymmetric, which may be exploited by Eve to steal information on the final key. For example, attacks that exploit the efficiency mismatch between two SPDs in the QKD system have been proposed, demonstrated, and analyzed; they include the time-shift attack [14-16] and the faked states attack [17]. Also, it has been demonstrated experimentally that Eve can tracelessly acquire the full secret key bits using specially tailored bright illumination [18]. Recently, a measurement-device-independent (MDI) QKD scheme was proposed to remove detector-side-channel attacks [19]. The advantage is that security can be guaranteed without regard to the experimental details of the measurement device. In this scheme, Alice and Bob send signal pulses to a Bell-state measurement device which may be owned by Eve and it is

\footnotetext{
*hua.lue@gmail.com

†chffung@hku.hk

${ }^{\ddagger}$ qycai@wipm.ac.cn
}

proven that they can distill some secure key bits based on the Bell measurement results.

Recently, the security of four-state deterministic quantum key distribution (DQKD) with a two-way quantum channel against the most general attacks has been proven by us $[20,21]$ under the ideal-device setting, while it is widely believed that two-way DQKD is vulnerable under Eve's practical attacks because Eve can attack the travel photon in both forward and backward lines. In this paper, we prove that two variations of the $\mathrm{DQKD}$ protocol are immune to all detector-side-channel attacks on Bob's side in the backward line. We do not analyze the MDI security of Alice's detectors and assume that they are ideal and nonblinded.

\section{SECURITY OF THE FOUR-STATE PROTOCOL}

In most $\mathrm{QKD}$ protocols, after distributing all signal qubits, Alice and Bob should communicate through public channel for basis reconciliation. In the BB84 protocol, about half of Bob's measurement results are discarded due to the inconsistent bases Alice and Bob used. ${ }^{1}$ But, in two-way DQKD protocols [23-28], Bob can decode Alice's key bits after measuring the returned qubits directly, without basis reconciliation. This means that all measurement results will be used for key distillation. ${ }^{2}$ The two-way DQKD was first proposed by using entanglement [23]. Later, a single-photon two-state protocol [26] was proposed for improving the experimental performance and then a single-photon four-state protocol was proposed independently in Refs. [27,28]. After that, the security of the two-way DQKD against some special attacks was studied $[24,29]$. Recently, the security of the four-state protocol against the most general attacks under the ideal-device setting was proved by us $[20,21]$.

Let us start with a brief review of the four-state two-way DQKD protocol with two encoding operations [see Fig. 1(a)].

\footnotetext{
${ }^{1}$ In the efficient BB84 protocol [22], the fraction of the measurement results to be discarded goes to zero asymptotically.

${ }^{2}$ This is true for any number of signals for the two-way protocol, not just asymptotically.
} 


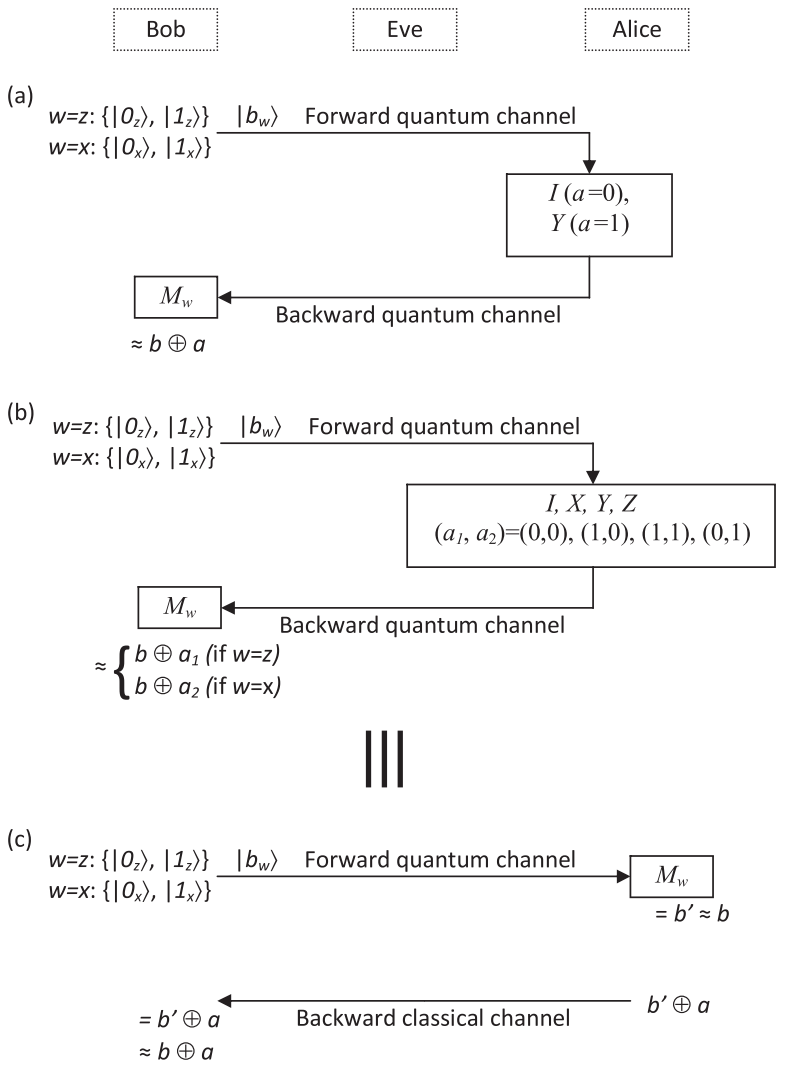

(BB84)

FIG. 1. We prove that the four-state DQKD protocols with two encoding operations (a) and four encoding operations (b) are MDI secure in line A-to-B. Protocol (b) was shown to be equivalent to protocol (c) where the forward channel runs the BB84 protocol and the backward channel runs a classical one-time pad (OTP) [21]. Here, $a, a_{1}$, and $a_{2}$ represent a raw secret key bit in all three protocols randomly chosen by Alice. In protocol (b), either $a_{1}$ or $a_{2}$ will be used as the key bit depending on the basis chosen by Bob which he will inform Alice of after his measurement. Here, $\left|b_{w}\right\rangle$ represents eigenstate $b$ of basis $w$ and $M_{w}$ represents a measurement in basis $w$.

(1) Bob prepares $n$ qubits randomly in one of the four states, $|0\rangle,|1\rangle,|+\rangle$, and $|-\rangle$, where $| \pm\rangle=(|0\rangle \pm|1\rangle) \sqrt{2}$, and sends them to Alice. (2) Alice randomly switches the communication to the check mode or the encoding mode. (3) In the check mode, Alice randomly measures part of the received states in the $X$ basis or the $Z$ basis. (4) In the encoding mode, Alice randomly performs the unitary operation $I=|0\rangle\langle 0|+| 1\rangle\langle 1|$ to encode bit 0 or $Y=|0\rangle\langle 1|-| 1\rangle\langle 0|$ to encode bit 1 . (5) Alice sends the encoded qubits back to Bob. Since Alice's encoding operations do not change the bases of Bob's states, i.e., $Y\{|0\rangle,|1\rangle\}=\{-|1\rangle,|0\rangle\}$ and $Y\{|+\rangle,|-\rangle\}=\{|-\rangle,-|+\rangle\}$, Bob measures each qubit in the same basis as the one he used for preparation to get Alice's key bit deterministically, without basis reconciliation. (6) After Bob measures all returned qubits, Alice announces her measurement results in the check mode to compute the fidelity of the forward states in the B-to-A channel for the consistent-basis measurements. They can get the fidelity $f_{0}, f_{1}, f_{+}$, and $f_{-}$of $|0\rangle,|1\rangle,|+\rangle$, and $|-\rangle$, respectively. (7) Alice announces part of her key bits in the encoding mode to compute the error-rate $e$ in the A-to-B channel. (8) Alice and Bob perform error-correction (EC) and privacy amplification (PA) to generate the final key bits. In the asymptotic scenario, after verifying $\xi \equiv$ $\left(f_{0}+f_{1}+f_{+}+f_{-}\right) / 2-1 \geqslant 1 / 2$, Alice and Bob can get the secure final key against general attacks with the generation rate

$$
r=1-h(\xi)-h(e),
$$

where $h(x)=-x \log _{2} x-(1-x) \log _{2}(1-x)$ is the binary entropy function, and $h(\xi)$ and $h(e)$ are the amounts of the key bits Alice and Bob should sacrifice for EC and PA.

With the idea of delaying PA, the security of a two-way DQKD protocol that uses four states and four encoding operations was proven [21] [see Fig. 1(b)]. It has been shown that the idea of delaying PA can simplify the security proof of two-way DQKD protocols, with the same key generation rate as that derived in Ref. [20]. The security of a two-way DQKD can also be proved against most general attacks with the entropic uncertainty relation, and a higher final key generation rate may be achieved [30].

\section{SECURITY OF THE FOUR-STATE PROTOCOL AGAINST ALL DETECTOR-SIDE-CHANNEL ATTACKS IN LINE A-TO-B}

We prove in this section the MDI security of line A-to-B for the original four-state protocol with two encoding operations proposed in Refs. [27,28], and the four-state protocol with four encoding operations proposed in Ref. [21]. Our proof relies on the use of equivalent protocols.

\section{A. Proof of MDI security of the four-state protocol with two encoding operations}

In order to prove the security of the MDI version of the protocol in line A-to-B, we modify the protocol by moving Bob's measurement to the hands of Eve in line A-to-B. The modified protocol is the same as the original protocol in Sec. II except with step (5) replaced as follows: (5) Alice sends the encoded qubit to Eve. Bob tells Eve the measurement basis. Eve makes a measurement on Alice's qubit using Bob's basis and returns the measurement result to Bob.

Note that Bob tells Eve the basis only after Alice has received the qubit on line B-to-A. This ensures that Eve cannot determine the initial qubit Bob sent out. Intuitively, we can understand the security of the modified protocol as follows. Since Bob randomly prepares the signal qubit in one of the four states, Eve cannot know Alice's encoding operations even if she gets Bob's measurement result. To know Alice's encoding, Eve has to know Bob's initial state as well.

To prove the security of the modified protocol rigorously, we consider the PA term in the key rate formula. The secure key rate $r_{\mathrm{PA}}$ from PA for the secret key generation is given by Renner and König's result [31]: $r_{\mathrm{PA}}=S\left(\rho^{A} \mid \rho^{B E}\right.$, basis $)=$ $S\left(\rho^{A B E} \mid\right.$ basis $)-S\left(\rho^{B E} \mid\right.$ basis $)$, where $\rho^{A B E}$ is the overall state of Alice's encoding $(A)$, the qubit emitted to line B-to-A $(B)$, and Eve's ancillas $(E)$, and $\rho^{B E}=\operatorname{tr}_{\mathrm{A}}\left(\rho^{A B E}\right)$. Note that system $B$ is released by Bob, encoded by Alice, and returned to Eve. This PA formula reflects that Eve, in order to learn about the key bit, uses the entire qubit from Alice and her own ancillas, which include those used in her attack on line B-to-A. This is consistent with the modified protocol where 
TABLE I. Key bit value dependence on the basis used by Bob ( $x$ or $z$ ) and Alice's encoding operation $(I, X, Y$, or $Z$ ). For instance, when Bob uses basis $x$, bit 1 is encoded by Alice if she applies $Z$ or $Y$ on the qubit emitted by Bob.

\begin{tabular}{lcc}
\hline \hline & \multicolumn{3}{c}{ Bit value } \\
\cline { 2 - 3 } Basis & 0 & 1 \\
\hline$x$ & $\{I, X\}$ & $\{Z, Y\}$ \\
$z$ & $\{I, Z\}$ & $\{X, Y\}$ \\
\hline \hline
\end{tabular}

Eve measures $\rho^{B E}$. We now show that $\rho^{A B E \mid \text { basis }}=\rho^{A B E}$; i.e., the overall state is not changed due to the knowledge of the basis.

Bob initially prepares the qubit $\rho^{B}$ in one of the four states. When the basis is $z$ or $x$, Bob prepares $\rho^{B \mid z}=\frac{1}{2}|0\rangle\langle 0|+$ $\frac{1}{2}|1\rangle\langle 1|$ or $\rho^{B \mid x}=\frac{1}{2}|+\rangle\left\langle+\left|+\frac{1}{2}\right|-\right\rangle\langle-|$, respectively. Since $\rho^{B \mid z}=\rho^{B \mid x}=I / 2$, further evolution of this state will be independent of the basis. Thus,

$$
\rho^{A B E \mid \text { basis }}=\rho^{A B E} .
$$

For completeness, we describe $\rho^{A B E}$ in more detailed as follows. Eve's most general attack on the B-to-A channel can be described as a joint unitary operation together with ancillas. After Eve's attack on the B-to-A channel, the joint state of the forward qubit and Eve's ancilla becomes $\rho_{B \rightarrow A}^{B E}=U_{B E}\left(\rho^{B} \otimes|E\rangle\langle E|\right) U_{B E}$, where $|E\rangle$ is the initial state of Eve's ancilla. After Alice's encoding operations, the joint state of backward qubit and Eve's ancilla becomes $\rho^{A B E}=$ $\frac{1}{2}|0\rangle\left\langle\left. 0\right|^{A} \otimes \rho_{0}^{B E}+\frac{1}{2} \mid 1\right\rangle\left\langle\left. 1\right|^{A} \otimes \rho_{1}^{B E}\right.$, where $\rho_{0}^{B E}=\rho_{B \rightarrow A}^{B E}$ and $\rho_{1}^{B E}=Y_{B} \rho_{B \rightarrow A}^{B E} Y_{B}$.

Therefore, Eq. (2) reduces $r_{\mathrm{PA}}$ to $r_{\mathrm{PA}}=S\left(\rho^{A B E}\right)-S\left(\rho^{B E}\right)$ for the modified protocol; and also $\rho^{A B E}$ in the modified protocol is the same as in the original protocol because $\rho^{B}$ undergoes the same evolution in both protocols. Therefore, we can directly apply the calculation of $r_{\mathrm{PA}}$ of the original protocol and its result to the modified protocol here. In summary, the modified protocol has the same key rate formula, Eq. (1). The four-state protocol is secure independent of the measurement and is immune against all detector-side-channel attacks in line A-to-B.

\section{B. Proof of MDI security of the four-state protocol with four encoding operations}

Here, we also show the MDI security in line A-to-B of the four-state protocol with four encoding operations of Ref. [21] [see Fig. 1(b)]. In this protocol, the steps are the same as the protocol given in Sec. II except with steps (4) and (5) replaced as follows: (4) In the encoding mode, Alice randomly performs unitary operations $I=|0\rangle\langle 0|+| 1\rangle\langle 1|, X=| 1\rangle\langle 0|+| 0\rangle\langle 1|$, $Z=|-\rangle\langle+|+|+\rangle\langle-|$, or $Y=|0\rangle\langle 1|-| 1\rangle\langle 0|$. The actual key bit value is dependent on the basis used (Table I). (5) Alice sends the encoded qubits back to Bob. Bob measures each qubit in the same basis as the one he used for preparation to get
Alice's key bit deterministically, without basis reconciliation. Bob also tells Alice his basis choice so that she knows the key bit value according to Table I.

We show that this protocol is MDI secure in line A-to-B. The security proof of this protocol given in Ref. [21] reduces the protocol to an equivalent protocol where the forward channel B-to-A runs the BB84 protocol and the backward channel A-to-B runs a classical one-time pad (OTP) (see Fig. 1(c) and Fig. 3 of Ref. [21]). It is argued in Ref. [21] that the classical OTP is equivalent to a quantum OTP by encoding classical states onto quantum states in some basis. The basis used has no bearing on the security since the security originates from that of the classical OTP: Eve is allowed to know the OTP-encrypted bit value in transit on line A-to-B. It is argued further in Ref. [21] that this basis is the basis used by Bob for his initial state. Thus, it is irrelevant whether Bob announces the basis before or after the OTP operation on line A-to-B. Using the same modification in Sec. III A for moving the measurement device from Bob to Eve, this variant of the four-state protocol is MDI secure in line A-to-B.

\section{DISCUSSION AND CONCLUSION}

Although the two-way DQKD has higher resistance to PNS attack-like attacks [32], decoy states are necessary to modify the four-state protocol for long-distance key distribution [33]. Another problem is that the two-way QKD is vulnerable to Eve's Trojan horse attacks, such as the invisible photon attack [29]. In practice, Alice can add a filter to defeat Eve's invisible photon attack. All Bob's photon pulses should pass through Alice's filter first and only wavelengths close to the operating wavelength are allowed to pass through.

It has been widely believed that, since Eve can attack the travel photons both in line B-to-A and in line A-to-B, QKD with a two-way quantum channel is more vulnerable under Eve's practical attacks compared with a one-way QKD protocol, such as the BB84 protocol. In this paper, we have shown that two four-state protocols are MDI secure in line A-to-B; i.e., when Eve instead of Bob measures Alice's qubit using Bob's basis, the modified protocols have the same key generation rate as that of the original protocols. Since line A-to-B is MDI secure, measurement-device-dependent attacks may only work in line B-to-A. Finally, we want to emphasize that we only proved the security of two-way DQKD against detector-side-channel attacks on Bob's side in the backward line A-to-B, while the MDI security of Alice's detectors in the check mode should be studied in future work.

\section{ACKNOWLEDGMENTS}

Financial support from NSFC under Grants No. 11104324 and No. 11074283 of the PRC government and the RGC under Grant No. 700712P of the HKSAR government is gratefully acknowledged.
[1] C. H. Bennett and G. Brassard, in Proceedings of the IEEE International Conference on Computers, Systems and Signal Processing, Bangalore, India (IEEE, New York, 1984), pp. 175-179.
[2] D. Mayer, J. ACM 48, 351 (2001).

[3] H.-K. Lo and H. F. Chau, Science 283, 2050 (1999).

[4] P. W. Shor and J. Preskill, Phys. Rev. Lett. 85, 441 (2000). 
[5] D. Gottesman, H. K. Lo, N. Lütkenhaus, and J. Preskill, Quantum Inf. Comput. 4, 325 (2004).

[6] N. Gisin, S. Fasel, B. Kraus, H. Zbinden, and G. Ribordy, Phys. Rev. A 73, 022320 (2006).

[7] C. Bennett, F. Bessette, G. Brassard, L. Salvail, and J. Smolin, J. Cryptol. 5, 3 (1992).

[8] S. Felix, N. Gisin, A. Stefanov, and H. Zbinden, J. Mod. Opt. 48, 2009 (2001).

[9] G. Brassard, N. Lütkenhaus, T. Mor, and B. C. Sanders, Phys. Rev. Lett. 85, 1330 (2000).

[10] N. Lütkenhaus, Phys. Rev. A 61, 052304 (2000).

[11] W.-Y. Hwang, Phys. Rev. Lett. 91, 057901 (2003).

[12] X.-B. Wang, Phys. Rev. Lett. 94, 230503 (2005).

[13] H.-K. Lo, X. Ma, and K. Chen, Phys. Rev. Lett. 94, 230504 (2005).

[14] B. Qi, C.-H. F. Fung, H.-K. Lo, and X. Ma, Quantum Inf. Comput. 7, 73 (2007).

[15] Y. Zhao, C.-H. F. Fung, B. Qi, C. Chen, and H.-K. Lo, Phys. Rev. A 78, 042333 (2008).

[16] C.-H. F. Fung, K. Tamaki, B. Qi, H.-K. Lo, and X. Ma, Quantum Inf. Comput. 9, 131 (2009).

[17] V. Makarov, A. Anisimov, and J. Skaar, Phys. Rev. A 74, 022313 (2006).

[18] L. Lydersen et al., Nat. Photon. 4, 686 (2010).

[19] H.-K. Lo, M. Curty, and B. Qi, Phys. Rev. Lett. 108, 130503 (2012).
[20] H. Lu, C.-H. F. Fung, X. Ma, and Q.-Y. Cai, Phys. Rev. A 84, 042344 (2011)

[21] C.-H. F. Fung, X. Ma, H. F. Chau, and Q.-Y. Cai, Phys. Rev. A 85, 032308 (2012).

[22] H.-K. Lo, H. F. Chau, and M. Ardehali, J. Cryptol. 18, 133 (2005).

[23] K. Boström and T. Felbinger, Phys. Rev. Lett. 89, 187902 (2002).

[24] A. Wójcik, Phys. Rev. Lett. 90, 157901 (2003); Q.-Y. Cai, ibid. 91, 109801 (2003).

[25] Q.-Y. Cai and B.-W. Li, Phys. Rev. A 69, 054301 (2004).

[26] Q.-Y. Cai and B.-W. Li, Chin. Phys. Lett. 21, 601 (2004).

[27] F.-G. Deng and G. L. Long, Phys. Rev. A 69, 052319 (2004).

[28] M. Lucamarini and S. Mancini, Phys. Rev. Lett. 94, 140501 (2005).

[29] Q.-Y. Cai, Phys. Lett. A 351, 23 (2006).

[30] N. J. Beaudry, M. Lucamarini, S. Mancini, and R. Renner, arXiv:1301.3138.

[31] R. Renner and R. König, in Second Theory of Cryptography Conference, TCC 2005, Vol. 3378 of Lecture Notes in Computer Science/Security and Cryptology, edited by J. Kilian (Springer, New York, 2005), pp. 407-425.

[32] M. Lucamarini and S. Mancini, arXiv:1004.0157.

[33] M. F. Abdul Khir, M. N. Mohd Zain, Iskandar Bahari, Suryadi, and S. Shaari, Opt. Commun. 285, 842 (2012). 